attendance on a therapeutic endoscopy course is not mandatory prior to going on to a GI bleed rota.

It is likely that trainees across the country may also feel inexperienced and ill equipped prior to starting on a GI bleed rota. We suggest that attendance on an emergency endoscopy therapeutics course should be a mandatory requirement for all gastroenterology trainees.

\section{P407 UK IBD STANDARDS: A ROADMAP TO IBD PHARMACY WORKFORCE TRANSFORMATION}

1,2Anja StClair Jones*, 2,3Uchu Meade. 'Brighton and Sussex University Hospitals NHS Trust, Brighton, UK; ${ }^{2} I B D U K$, London, UK; ${ }^{3}$ St. Mark's Hospital, London, UK

\subsection{6/gutjpl-2020-bsgcampus.481}

Background Inflammatory Bowel Disease (IBD) Specialist Pharmacy Services (SPS) are currently not embedded in UK IBD services and therefore neither recognised by policy makers nor commissioned by service providers. We report on the incorporation of SPS into national standards and the e-benchmarking tool to drive pharmacy workforce transformation in IBD.

Method A multidisciplinary alliance of 17 organisations and patients (IBDUK) was convened to update the current UK IBD standards. To inform the development of medicines optimisation related standards, IBD units with developed SPS were informally surveyed. The pharmacy representatives determined quantity and quality of advanced practice based on published IBD service descriptions, SPS feedback and the Royal Pharmaceutical Society (RPS) Framework for Advanced Practice (APF).

An e-Delphi consensus process was undertaken over 3 rounds by IBDUK to refine a set of evidence- and expert opinion-based recommendations for optimal service delivery across the patient journey with $80 \%$ agreement required for statements to be retained.

These agreed standards informed the benchmarking tool to enable self-assessment supporting quality improvement and additional resources requests where needed. Descriptors were developed in 2 consensus workshops by IBDUK with expert pharmacy representation.

Results IBDUK agreed 59 standards in total with $4(7 \%)$ describing SPS which were incorporated with $100 \%$ agreement.

To enable workforce transformation 3 transformative drivers were considered: Capability is described in all 4 IBD SPS standards outlining pharmacy leadership, medicines expert roles and Multidisciplinary Team (MDT) working from diagnosis to long-term care using the RPS APF to provide a clear development pathway. Motivation is supported through professional recognition requiring an expert pharmacist RPS Faculty Stage 2 as a core-member of the MDT and Opportunity for advance practice is provided through mandating 0.6 whole time equivalent of expert pharmacist in IBD per 250,000 population.

The benchmarking tool developed and agreed by IBDUK to drive quality defines A-D descriptors for all standards, demonstrating $\mathrm{A}=$ 'excellent, proactive' to $\mathrm{D}=$ 'minimal, inadequate' care. RPS standards and SPS feed back were used to define descriptors relevant to pharmacy.

Conclusion IBDUK and RPS Faculty describe a road map for pharmacy workforce development in IBD and mandates units to develop SPS.

UK IBD Standards 2019 for the first time embed and describe SPS as an integral part of the IBD MDT managing IBD patients and enables recognition and commissioning of expert pharmacy practice. 